\title{
Selección e implementación de Sistemas de Información Contable y Administrativo: una herramienta para la competitividad de las Mipymes
}

\author{
Diego Alejandro Peralta Borraya \\ Fabiola Loaiza Robles ${ }^{b}$
}

Información del artículo Recibido: 19 de diciembre/2017 Aceptado: 15 de marzo/2018

Clasificación JEL M15

Este es un artículo Open Access bajo la licencia CC BY NC [https://creativecommons.org/licenses/by-nc/4.0]

Enlace DOI

https://dx.doi.org/10.24142/rvc.n16al

\section{Sugerencia de citación}

- Peralta Borray, D. A. \& Loaiza Robles, F. (2017). Selección e implementación de Sistemas de Información Contable y Administrativo: una herramienta para la competitividad de las Mipymes. Revista Visión Contable, 16, 10-29. doi: 10.24142/rvc. n16al

\begin{abstract}
Selection and implementation of Accounting and Administrative Information Systems: a tool for the competitiveness of MSMEs
\end{abstract}

\section{Resumen}

Contar con información oportuna y relevante es un factor clave en la competitividad de una Mipyme; se ha demostrado ampliamente que contar con un sistema de información contable y administrativo (SICA) apropiado para la organización mejora su capacidad de adaptación y reduce los costos de oportunidad derivados de las asimetrías de información. Adicionalmente, la implementación de un sistema acorde al negocio crea procesos de habituación que reestructuran las relaciones entre las líneas jerárquicas, mejora la coordinación y comunicación, y permite establecer medidas claras de desempeño.

Este documento busca identificar los elementos centrales que deben tenerse en cuenta al momento de seleccionar e implementar un SICA, con el fin de obtener los mayores beneficios de estas actividades.

Palabras clave:

Sistemas de Información Contable, Competitividad, Información Contable y Administrativa.

\begin{abstract}
Having timely and relevant information is a key factor in the competitiveness of a Mipyme, it has been widely demonstrated that having an accounting and administrative information system (AAIS) appropriate for the organization improves its ability to adapt and reduces the opportunity costs derived of information asymmetries. Additionally, the implementation of a system according to the business creates processes of habituation that restructure the relations between the hierarchical lines, improve coordination and communication and; they allow to establish clear performance measures.

This document seeks to identify the central elements that must be considered when selecting and implementing a AAIS, in order to obtain the greatest benefits from these activities.
\end{abstract}

\section{Key words:}

Accounting Information Systems, Competitiveness, Accounting and Administrative Information.

a Contador Público y Magíster en Administración de Empresas de la Universidad Nacional de Colombia. Profesor de la Facultad de Ciencias Administrativas y Contables de la Universidad de La Salle. Integrante del grupo de investigación "SIGMA empresarial". Correo electrónico: daperalta@unisalle.edu.co.

b Contadora pública de la Universidad de Manizales, Colombia. Magíster en Educación y Desarrollo Humano del Centro Internacional de Educación y Desarrollo Humano (Cinde) y la Universidad de Manizales. Docente de tiempo completo en la Universidad de La Salle, Bogotá, Colombia. Correo electrónico: floaiza@unisalle.edu.co. 


\section{Introducción}

La competitividad para una Mipyme depende de múltiples factores externos, como el sector, la infraestructura regional o del país, las condiciones de demanda, el acceso a mano de obra calificada, etc. Sin embargo, estos, en su mayoría, no son controlables por las Mipymes, que entre más pequeñas tienen una menor posibilidad para influir o asegurarse ante los riesgos derivados de los mismos. Por ende, la búsqueda de la competitividad debe enfocarse en optimizar las variables internas; es decir, aquellas que pueden ser intervenidas directamente, lo cual no se refiere a dejar de analizar los factores externos, sino que implica generar capacidades que permitan obtener flexibilidad y capacidad de reacción ante cambios del entorno.

FIGURA 1. Factores internos que afectan la competitividad. Adaptado de Saavedra García (2012).
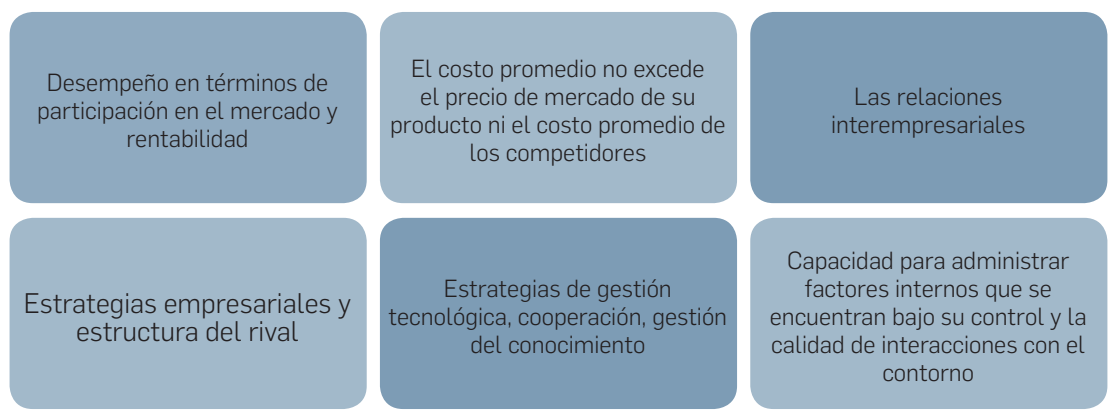

Capacidad para administrar factores internos que se encuentran bajo su control y la calidad de interacciones con el contorno



Sostenido crecimiento de la productividad

Se ha demostrado que la implementación de un sistema de información contable y administrativo (SICA) afecta las variables indicadoras de la competitividad interna de la empresa, mejora la eficiencia y la gestión; por lo que no es de extrañar que los "sistemas de información" hagan parte del 
mapa de competitividad del Banco Interamericano de Desarrollo (BID), ${ }^{1}$ en donde, como menciona María Saavedra (2012), "el apoyo a las funciones operativas a través de las tecnologías de información resulta de vital importancia para desarrollar una estructura competitiva del negocio (Katz \& Hubert, 2003; RICYT, 2009 y Martínez \& Alvarez, 2006)” (p. 106). La implementación, cambio o ajuste del sistema de información incide, por lo menos, en dos de los factores generadores de competitividad interna. Por lo tanto, la selección o diseño de un SICA para el negocio se convierte en una acción estratégica.

No obstante, un estudio realizado por Mora Riapira, Vera Colina y Melgarejo Molina (2015), el cual evaluó la competitividad de las Mipymes del sector comercio en Bogotá, encontró que "las microempresas tienen niveles de competitividad preocupantes en las áreas de recursos humanos, gestión ambiental y sistemas de información" (p. 85). Este estudio también identificó una gran diferencia entre las Pymes que exportan y las que no:

se puede identificar que los índices de competitividad de las exportadoras es mayor [sic] a las no exportadoras, encontrando una diferencia significativa en la dimensión de sistemas de información (17\%) que se evidencia con la necesidad de contar con información en los mercados dinámicos que exigen un nivel de respuesta inmediato. (Mora Riapira, Vera Colina, \& Melgarejo Molina, 2015, p. 85).

Como puede observarse, la correcta implementación de un SICA puede convertirse en un factor clave para la competitividad. Esta postura se refuerza por los estudios realizados por Laudon y Laudon (2008), citados por Guerrero Julio y Gómez Flórez (2012):

El auge en el desarrollo de los sistemas de información ha generado mayor crecimiento y competitividad en las organizaciones al apoyar los procesos de negocio, las actividades de procesamiento de la información y las actividades

\footnotetext{
"El 'Mapa de Competitividad' fue desarrollado por el Banco Interamericano de Desarrollo (BID) y adaptado por la Cámara de Comercio de Medellín y Antioquia para ser utilizado en el diagnóstico organizacional de la pyme; visualiza a la organización como un sistema integrado por nueve áreas que al interconectarse entre sí mejoran el desempeño de la organización" (Martínez \& Álvarez, 2006, como se citó en Saavedra García, 2012, p. 106); las áreas a las que hace referencia son: Planeación estratégica; Calidad, producción y operaciones; Comercialización; Contabilidad y Finanzas; Recursos Humanos; Gestión ambiental; y Sistemas de información.
} 
de administración, lo que abre un sinnúmero de posibilidades para ampliar las relaciones entre clientes, proveedores y empleados, y posibilita la rapidez en las respuestas a los cambios en el entorno. (p. 88).

En este sentido, "el cambio en los sistemas de información se vuelve imprescindible para apoyar la respuesta de las organizaciones a su entorno, de tal manera que se convierta en un dispositivo que transmita la necesidad de llevar a cabo transformaciones organizativas" (Vanegas Nieto, García Pérez de Lema, \& Marín Hernández, 2009). Por otra parte, los sistemas de información administrativa y contable pueden propiciar la innovación, la implementación de un sistema; ya sea a través del diseño a la medida o su selección para la organización, es ya una innovación en los procesos administrativos. Como mencionan Vanegas Nieto et al (2009), "desde el punto de vista empírico, Bisbe y Otley (2004), con una muestra de 120 empresas españolas, demostraron que a mayor uso de SCG, mayor es el efecto de la innovación en el rendimiento de la pyme” (pág. 218)

\section{Sistemas de Información Contables y Administrativos}

Una de las primeras decisiones de las que tiene que ocuparse la dirección de una Mipyme es la adquisición de un software de contabilidad o un sistema que lleve la nómina; más adelante y de acuerdo al tipo de operación, se evalúa la implementación de un sistema de información para el área de ventas o de un sistema de costos.

Normalmente, la licencia para el paquete de contabilidad se adquiere por la presión del profesional en contabilidad de la empresa, quien ya conoce la operación de un paquete en específico, bien sea porque lo ha usado en otras organizaciones o porque es el que aprendió en la universidad. Es común que el principal objetivo de esta compra sea agilizar el procesamiento de la información contable con miras a la declaración y pago de los impuestos. "La condición general de las PyMES es su baja utilización de la Tecnología Informática, y el uso de los sistemas contables con fines eminentemente fiscales, dejando de lado la función de la información como base en la toma de decisiones" (Astudillo Cerón, 2008, pág. 53).

En raras oportunidades se decide contratar el diseño de un sistema de información contable a la medida; es decir, desarrollado teniendo en cuenta la estructura administrativa y necesidades de información del negocio. 
Sin embargo, para el caso del sistema de información de costos o el de gestión de ventas, es más probable que la gerencia decida contratar un desarrollo a la medida.

En todo caso, el diseño o selección de un sistema de información apropiado para la organización se toma a la ligera, considerando que es una gran oportunidad para optimizar e innovar en los procesos administrativos, estandarizar los productos de los empleados administrativos de nivel operativo, contar con información para alinear a los gerentes medios o profesionales vitales con la estrategia de negocio, contar con información para evaluar cursos de acción y minimizar los costos de oportunidad que generan las decisiones administrativas.

Como lo presentan Burns y Scapens (2000, como se citó en Facin Lavarda, Ripoll Feliu y Barrachina Palanca, 2009), "un SCG puede ser analizado como una institución dentro de una organización, dado que tiene la capacidad de difundir y asentar en esta un conjunto de reglas y rutinas que pueden cambiar a través del tiempo" (p. 103). Por lo tanto, es posible que el sistema de información cree y reproduzca nuevas rutinas, nuevas normas diarias, para finalmente institucionalizarlas.

El efecto de la implementación de los sistemas de información en la cultura de la organización se había advertido desde hace bastante tiempo. Ya en 1983, Flamholtz (como se citó en Vanegas Nieto et al., 2009), indicaba que "la cultura y la estrategia de la empresa están fuertemente relacionadas con la implantación de un SCG. Estos sistemas se utilizan para transmitir y reforzar la cultura de las empresas a través de la organización y de manera estratégica para la toma de decisiones" (p. 217).

En el estudio realizado por Facin Lavarda et al. (2009), se muestra que la implementación de un sistema contable de gestión permitió "solucionar problemas que la gerencia tenía con la falta de información oportuna y fiable, sin romper con las rutinas organizativas, problemas como costes innecesarios y un elevado esfuerzo para elaborar información para la toma de decisiones" (p. 114). Como puede observarse, una de las ventajas principales de implementar un sistema de información contable es que este proceso permite modificar la cultura de la organización, y esta capacidad, aprovechada correctamente a través de una detallada planeación sobre lo que queremos que ocurra en las dinámicas organizacionales, es un factor clave para lograr alinearse con la estrategia.

Adicionalmente, la implementación de un sistema de información que apoye no un gran número, sino la totalidad de procesos administrativos, 
ayudará a abordar algunas de las problemáticas clave de las Pymes identificadas por Zeballos ${ }^{2}$ (2007, como se citó en Saavedra García, 2012), en especial las de "ausencia de cooperación, la desconfianza, la 'vocación' por actuar aisladamente" (p. 97).

También es importante añadir que contar con información para tomar decisiones acertadas implica ver las posibilidades que de otra forma no serían claras para el administrador, por lo que se reduce el riesgo de costo de oportunidad. Como bien lo expresa Barreiro Noa (2003), "la empresa asume el costo de oportunidad de la información, en los ingresos que deja de percibir si no utiliza las variantes que la información brinda, y aquí sí se concreta el verdadero costo de oportunidad de la información y el conocimiento" (p. 29).

Por otro lado, el uso de diferentes Tecnologías de la Información y la Comunicación (TIC) mejora la percepción de los gerentes con relación al rendimiento del negocio, creando la necesidad de lograr una correcta articulación entre el sistema de información y otras TIC. Acerca de los efectos de las tecnologías de información y comunicación sobre el rendimiento en procesos internos, Gálvez Albarracín, Riascos Erazo y Contreras Palacios (2014) encontraron, al estudiar 1201 Mipymes, que "la disponibilidad y utilización de las diferentes herramientas y prácticas TIC en ambiente web hace que mejoren la calidad de los productos de la empresa, así mismo que sus procesos internos sean más eficientes" (p. 361). En este mismo estudio se plantea que "la disponibilidad y la utilización de las diferentes herramientas y prácticas de TIC en ambiente web al interior de la Mipyme contribuyen a que sus colaboradores estén más satisfechos y a que se reduzca el ausentismo laboral" (p. 362).

\section{Diseño, selección o contratación de un sistema de información que aporte a la competitividad del negocio}

La primera disyuntiva estriba en si es preferible: 1) comprar la licencia de un sistema de información; 2) contratar un outsourcing que se encargue de satisfacer los requerimientos de información del negocio (por ejemplo, una firma de contadores o auditores que ya cuente con las licencias); o 3) diseñar-

Este trabajo estudia las Pymes latinoamericanas de ocho países, entre ellos Colombia. 
lo de acuerdo a la estrategia del negocio. Para resolver el dilema es pertinente observar que comprar un sistema de información estándar es más económico que contratar su elaboración, a la medida, a una empresa especializada o vincular desarrolladores al negocio para que realicen esta labor.

El desarrollo a la medida permitirá que la dirección de la empresa establezca puntos claros de direccionamiento y control sobre los procesos y procedimientos de la organización, lo que con un adecuado trabajo de planeación la alinearía por completo con la estrategia de negocio. Se debe recordar que, en ambos casos, requerirá de un proceso de planeación detallado que le obligará a modificar sus procesos y procedimientos. Cualquier modificación que se realice a los mismos debe evaluarse en la óptica de la creación de valor para la empresa.

Con relación a la contratación de un outsourcing para el sistema de información, esta opción es la menos recomendable, aunque, como defienden algunos autores, permite a la organización centrarse en temas estratégicos, aumenta la flexibilidad en la renovación del hardware y se obtiene una reducción en costos de personal y tecnología. Por otro lado se encuentran los riesgos, como los mencionados por González Ramírez, Gascó Gascó y Llopis Taverner (2010), que incluyen la falta de cumplimiento del proveedor, el no entendimiento de las necesidades del cliente, la dependencia que genera este servicio, la pérdida del conocimiento técnico de la empresa y la incapacidad del proveedor para adaptarse a nuevas tecnologías; factores que opacan las posibles ventajas.

\section{Contratar el diseño de un sistema de información}

Si se opta por el diseño a la medida del sistema de información, debe tenerse en cuenta que no se requieren sistemas de información complejos, sino funcionales y acordes a lo que se planea en términos de cultura y estrategia. En muchos casos es más económico y funcional diseñar una aplicación basada en Microsoft Excel o Access que embarcarse en el desarrollo bajo un lenguaje más complejo como SQL, JAVA, ORACLE, VISUAL, etc.

No obstante, un sistema desarrollado bajo un lenguaje avanzado le permitirá a la empresa la interacción con otras TIC; por ejemplo, tener acceso a sus bases de datos e información financiera en tiempo real a través de la web e, incluso, desde su celular a partir de una App. Adicionalmente, la cantidad de datos que puede manejar es mayor a la capacidad de Excel o 
Access, y es posible crear o modificar accesos, bases de datos y procesos, permitiéndole al sistema crecer junto con las necesidades de la organización. Como desventaja, sin duda, se encuentra el costo del diseño del sistema y el mantenimiento del mismo.

Por otro lado, si se decide vincular desarrolladores al negocio, es posible que se impulsen nuevas capacidades internas; sin embargo, esta opción debe revisarse con cuidado: la evaluación de los niveles de ocupación que tendrán los nuevos miembros de la organización una vez terminado el desarrollo y la posibilidad de usar los conocimientos apropiados en otras áreas de la organización son factores importantes a la hora de una posible vinculación.

\section{Selección de un sistema de información}

Si las barreras de costo de desarrollo de un sistema a la medida exceden las posibilidades del negocio, la alternativa está en la compra de un software estándar; en este caso, la selección del sistema apropiado dependerá de varios factores:

FIGURA 2. Factores a tener en cuenta en la selección del sistema. Elaboración Propia.

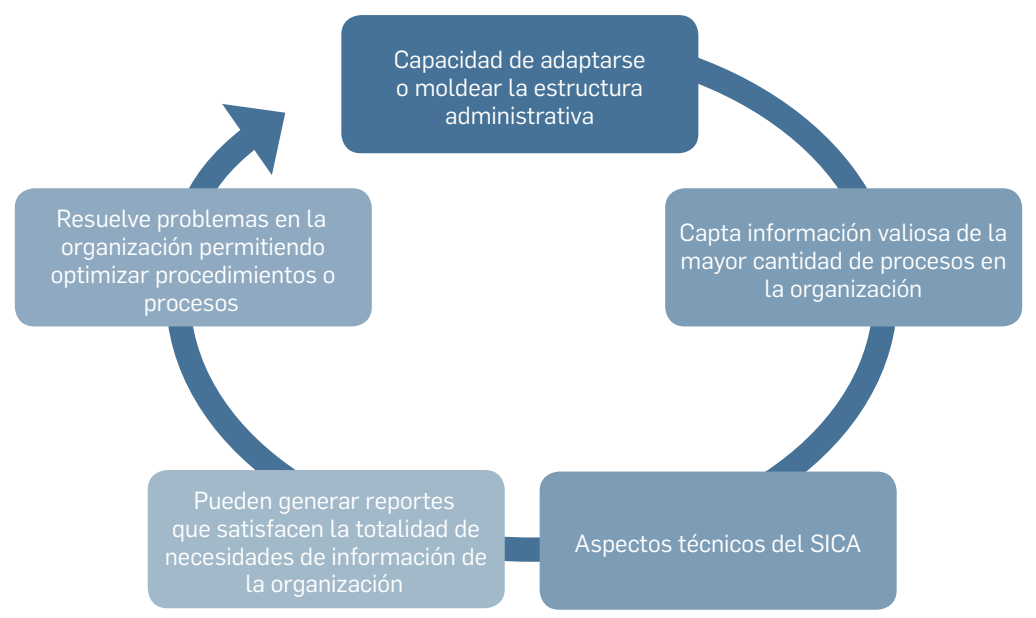




\section{1 Capacidad de adaptarse o moldear la estructura administrativa}

Debe observarse cuál es el tipo de estructura organizativa que tiene la empresa, esto incluye revisar cómo están definidas las líneas jerárquicas; los manuales de funciones y procedimientos; y los procesos de toma de decisiones. Es importante, además, tener en cuenta que algunos de los sistemas de información permiten editar los accesos y actividades para otorgar permisos de acceso a las pantallas de captura o reporte de información. Sin embargo, existen otros que tienen niveles de acceso para los usuarios de forma estándar, y en estos casos debe preverse que al implementar el sistema será necesario ajustar los manuales de procedimientos y funciones de las personas que tendrán relación directa con él.

Por ejemplo, si la actividad "elaborar el reporte de cartera" actualmente es desarrollada por el auxiliar contable, pero los accesos del sistema la diferencian de otra de las actividades a cargo del auxiliar que es "facturación", con el objetivo de adaptarse al sistema debe establecerse un responsable de cada tipo de acceso, obligando a modificar el procedimiento y las funciones de los empleados. Para evaluar esta correspondencia se propone usar herramientas como la siguiente:

\begin{tabular}{|c|c|c|c|c|}
\hline Cargo & $\begin{array}{l}\text { Actividades } \\
\text { (ingreso, } \\
\text { modificación o } \\
\text { extracción de } \\
\text { información) }\end{array}$ & $\begin{array}{l}\text { Procedimiento } \\
\text { al que } \\
\text { pertenece la } \\
\text { actividad }\end{array}$ & $\begin{array}{l}\text { Nivel de acceso } \\
\text { que se puede } \\
\text { otorgar en el } \\
\text { sistema }\end{array}$ & $\begin{array}{l}\text { Actividad } \\
\text { operativa o } \\
\text { decisional }\end{array}$ \\
\hline \multirow{2}{*}{$\begin{array}{l}\text { Auxiliar } \\
\text { Contable }\end{array}$} & $\begin{array}{c}\text { Diligenciamiento } \\
\text { de la factura de } \\
\text { venta }\end{array}$ & Facturación & Nivel I & Operativa \\
\hline & $\begin{array}{l}\text { Elaborar el } \\
\text { reporte de } \\
\text { cartera. }\end{array}$ & $\begin{array}{l}\text { Control de } \\
\text { cartera }\end{array}$ & Nivel II & Operativa \\
\hline
\end{tabular}

Nota: Elaboración propia. 
Como puede observarse, existen dos alternativas para la gerencia: una es adaptar los procesos y procedimientos de acuerdo a la funcionalidad del sistema sin modificar los accesos estándar, y la otra es planear una estructura organizacional óptima y ajustar los accesos del sistema a esta planeación. En ambos casos se logran beneficios de la implementación del SICA; en el primero es factible organizar los procesos y procedimientos que muchas veces no están definidos claramente, ya que no todas las Mipymes cuentan con manuales de funciones y procedimientos serios. En la segunda opción es más fácil controlar el despliegue de la estrategia; sin embargo, requ erirá de un mayor esfuerzo por parte de la dirección, ya que debe rediseñar la estructura administrativa del negocio.

Si se modifican los accesos para que sean exactamente iguales al funcionamiento actual del negocio, la adquisición e implementación del sistema no producirá cambios en la organización y, por ende, la empresa no ganará en términos de competitividad interna por esta vía.

\section{2 Resuelve problemas en la organización permitiendo optimizar procedimientos o procesos}

Partiendo del ideal sobre el funcionamiento de la organización, debe realizarse un diagnóstico detallado de las ineficiencias de la organización en el campo administrativo, realizando listas de chequeo sobre los problemas que más se repiten en lo rutinario y el impacto que estas dificultades han tenido en el desempeño del negocio; por ejemplo, si se han presentado dificultades en la facturación o compra de insumos, debe detallarse claramente a qué procedimiento pertenecen, quién es el responsable y cuáles son las causas probables del problema. Lo anterior permitirá evaluar qué actividades podrían ser optimizadas con el uso del sistema que se está evaluando. (Ver Tabla 2)

\section{3 Capta información valiosa de la mayor cantidad de procesos en la organización}

En este punto deben considerarse cuáles son los insumos de información valiosa para la organización; por ejemplo, la facturación, los comprobantes de egreso, extractos bancarios, hojas de ruta de costos (para el caso de empresas de producción de bienes o servicios), directorios de clientes, etcétera. Es importante validar, además, si el sistema a adquirir permite ingresar 


\begin{tabular}{|c|c|c|c|c|c|}
\hline $\begin{array}{l}\text { Descripción del } \\
\text { problema }\end{array}$ & $\begin{array}{l}\text { Impacto en } \\
\text { el negocio }\end{array}$ & $\begin{array}{l}\text { Procedimien- } \\
\text { to al que } \\
\text { pertenece el } \\
\text { problema }\end{array}$ & $\begin{array}{l}\text { Responsable del } \\
\text { procedimiento o } \\
\text { actividad }\end{array}$ & $\begin{array}{l}\text { Causas } \\
\text { probables }\end{array}$ & $\begin{array}{l}\text { El SICA } \\
\text { resuelve el } \\
\text { problema } \\
\text { identificado }\end{array}$ \\
\hline $\begin{array}{l}\text { No hay claridad } \\
\text { sobre los valo- } \\
\text { res, cantidades } \\
\text { y referencias } \\
\text { compradas por } \\
\text { los clientes, } \\
\text { impidiendo su } \\
\text { clasificación y } \\
\text { aplicación de } \\
\text { tarifas diferen- } \\
\text { ciales, cupos de } \\
\text { crédito, etc. }\end{array}$ & $\begin{array}{l}\text { (Alto/Medio/ } \\
\text { Bajo) }\end{array}$ & Facturación & Emma Granados & $\begin{array}{l}\text { No existe } \\
\text { reporte de } \\
\text { información } \\
\text { consolidada de } \\
\text { las ventas por } \\
\text { cliente. } \\
\text { Hay dificultades } \\
\text { en la digitación } \\
\text { de los NIT de } \\
\text { los clientes. }\end{array}$ & $\begin{array}{l}\text { (SI/NO/ } \\
\text { Parcialmente) }\end{array}$ \\
\hline \multicolumn{6}{|c|}{ Nota: Elaboración propia. } \\
\hline
\end{tabular}

la mayor cantidad de información clasificada como importante para la empresa, pues "de nada sirve una herramienta que cubra unas pocas operaciones y no proporcione soporte a la totalidad de las necesidades" (Astudillo Cerón, 2008, pág. 53.

\section{TABLA 3. Evaluación de la capacidad del SICA para captar información valiosa}

Información valiosa

Información sobre cantidades y valores facturados a los clientes en cada período

Información sobre fechas de cumplimiento y valor de las obligaciones (pagos a terceros)

Nota: Elaboración propia.

(1)

Esta información puede incorporarse al software
(Sí/No/Parcialmente)
Parcialmente (incorpora valores, Compras pero no fechas de vencimiento)
$\begin{array}{ll} & \text { Facturación } \\ & \\ \text { valores, Compras } \\ \text { miento) }\end{array}$

\section{(Si/N}

Procedimiento asociado

Si existen otros sistemas de información en la organización, debe verificarse la posibilidad de migrar los datos al nuevo sistema. Esta actividad es normalmente sencilla a través de la obtención de archivos de texto; sin embargo, cuando se trata de sistemas antiguos o no se cuenta con esas capacidades en los empleados de la empresa, se sugiere contratar un experto que realice la migración y garantice la integridad de los datos. 


\section{4 Capacidad del SICA para generar reportes acordes a las necesidades de la empresa}

Es importante evaluar la capacidad del sistema para producir la totalidad de los reportes que la gerencia requiera para el despliegue eficaz de la estrategia y la toma de decisiones rutinarias. Lo anterior también es mencionado por Vanegas Nieto et al. (2009) de la siguiente manera: el "SCG debe producir cualquier tipo de información que sea requerida por el administrador en la implementación de la estrategia más eficaz para su organización” (p. 217).

Por lo tanto, sería ideal que el sistema contara con un módulo de parametrización o desarrollo de reportes que pueda ser operador por una persona con conocimientos mínimos en sistemas; de lo contrario, la solicitud a la compañía que desarrolló el software de un nuevo reporte hecho con base en las necesidades de la empresa puede generar costos excesivos. Por lo anterior, se recomienda la utilización de una lista de chequeo que permita identificar si el sistema a adquirir cumple con la totalidad de las funciones de reporte requeridas.

\section{TABLA 4. Evaluación del cumplimiento de las necesidades de información}

\begin{tabular}{l|l|l|l|l}
\hline $\begin{array}{l}\text { Detalle del } \\
\text { requerimiento } \\
\text { de información }\end{array}$ & $\begin{array}{c}\text { Este informe } \\
\text { es estándar en } \\
\text { el SICA }\end{array}$ & $\begin{array}{c}\text { El sistema cuenta } \\
\text { con un editor de in- } \\
\text { formes que permite } \\
\text { obtener esta infor- } \\
\text { mación sin causar } \\
\text { altos costos }\end{array}$ & $\begin{array}{c}\text { Procedimien- } \\
\text { to asociado al } \\
\text { informe }\end{array}$ & $\begin{array}{c}\text { Cargo que } \\
\text { requiere } \\
\text { y usará el } \\
\text { reporte }\end{array}$ \\
\hline $\begin{array}{l}\text { Reporte sobre la } \\
\text { cartera vencida } \\
\text { que indique el } \\
\text { tiempo de mora } \\
\text { de la factura, } \\
\text { el valor, la } \\
\text { razón social } \\
\text { del cliente, los } \\
\text { antecedentes de } \\
\text { mora del cliente } \\
\text { y su calificación } \\
\text { en el sistema } \\
\text { de gestión de } \\
\text { clientes }\end{array}$ & Sí & Cobranzas & Jefe de \\
Cobranzas
\end{tabular}




\section{5 Aspectos técnicos sobre el SICA}

Es vital que la empresa realice un análisis costo-beneficio para determinar los requerimientos técnicos mínimos del sistema de información. Como ya se mencionó, en muchos casos es más económico y funcional diseñar una aplicación basada en Microsoft Excel o Access que embarcarse en la implementación de un sistema de información basado en un motor de base de datos.

Sin embargo, debe tenerse cuidado en esta decisión, porque al crecer la operación del negocio, van a requerirse interacciones de varios usuarios al mismo tiempo en el sistema, así como el almacenamiento de una mayor cantidad de registros, será necesario contar con interacciones del sistema con otras TICs, incluyendo la necesidad de consultas e ingreso de información vía web, por lo que es preferible adquirir o diseñar un sistema que use un estándar de almacenamiento adecuado. Al respecto, Astudillo Cerón (2008) define cuáles son los mejores productos del mercado (Tabla 5).

\begin{tabular}{l|l}
\hline \multicolumn{2}{|c|}{ TABLA 5. Mejores estándares de almacenamiento } \\
\hline \multicolumn{1}{|c|}{ COMERCIALES } & DE USO LIBRE \\
\hline Oracle Data Base Server & PostgreSQL \\
\hline Microsoft SQL Server & SAP DB (MaxDB) \\
\hline IBM DB/2 & MySQL Server \\
\hline Nota: Adaptado de Astudillo Cerón (2008). \\
\hline
\end{tabular}

Al seleccionar el SICA se deben tener en cuenta los siguientes elementos:

- Debe estar desarrollado con tecnología para su acceso desde la web.

- Debe soportar multiusuario, es recomendable que permita el acceso ilimitado de usuarios.

- Si en algún momento se interrumpe el funcionamiento del sistema, los datos no deben verse alterados. 
- Debe permitir procedimientos de seguridad de la información, como copias regulares de seguridad de los datos y control de usuarios que modifican la información.

\section{Planeación de la implementación}

La implementación de un sistema de información contable y de gestión requiere no solamente la instalación del paquete en los ordenadores, sino de un proceso regulado que comienza con la identificación y gestión de los riesgos relacionados con la implementación y operativización del sistema. A este respecto es pertinente mencionar el modelo presentado por Guerrero Julio y Gómez Flórez (2012):

FIGURA 3. Modelo de gestión de riesgos y controles en sistemas de información. Adaptado de Guerrero Julio y Gómez Flórez (2012).

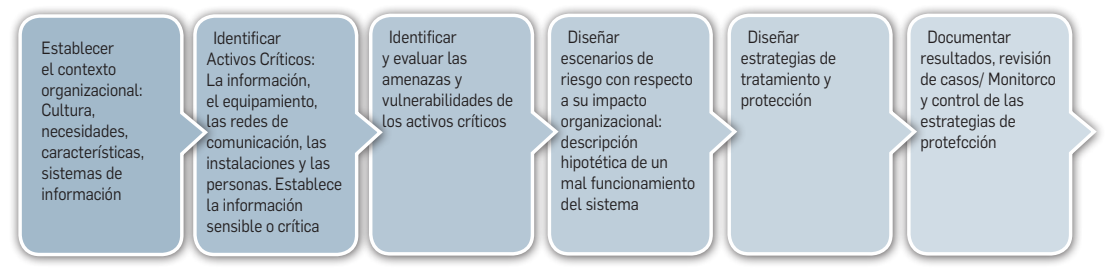

Este modelo propone una serie de pasos para gestionar el riesgo y establecer controles sobre el sistema de información, los cuales se abordarán brevemente con el objetivo de contextualizarlos y adaptarlos en la actividad de implementación que ocupa el presente trabajo.

- Paso 1: se requiere establecer los sistemas de información existentes en el negocio y cómo estos aportan a la estructura organizativa. En este paso se recomienda realizar entrevistas a los usuarios del o los sistemas, con el fin de identificar los riesgos que existen actualmente y los que podrían generarse al retirar estas aplicaciones para dar paso al nuevo sistema de información. 
- Paso 2: se sugiere 1) la realización de un inventario de los activos relacionados con los sistemas de información (física e intangible); 2) determinar qué información es sensible o crítica; y 3) detectar las posibles vulnerabilidades. Esta actividad puede ser alimentada con el paso anterior, sugerido para la selección del sistema en donde se identificó la información considerada como valiosa para la organización. En este punto deben determinarse las salidas de los otros sistemas para exportar la información, archivos txt, importaciones a Excel, etc. Debe determinarse si es posible migrar la totalidad de la información de los sistemas existentes al nuevo software.

- Paso 3: consiste en elaborar los escenarios de riesgo de la implementación, los que pueden referirse a 1) la operación del sistema; 2) los posibles fallos en cuanto a la disponibilidad, autenticidad, integridad y confidencialidad de la información; o 3) los riesgos inherentes a los controles administrativos existentes derivados del sistema de control interno de la empresa.

- Paso 4: establecer estrategias para mitigar los riesgos identificados. Estas pueden requerir ajustar el sistema o las políticas de control interno, y deben analizarse por escenarios y seleccionarse de acuerdo a la probabilidad de ocurrencia y el criterio de la gerencia. Por último, debe establecerse un plan para el desarrollo de las actividades de mitigación del riesgo seleccionadas (estas pueden ir desde la modificación de un procedimiento hasta la revaluación de la política de compras de la compañía).

- Paso 5: establecer acciones y responsables del monitoreo constante de la operación del sistema una vez se haya implementado.

\section{Implementación del sistema}

Para la implementación del SICA debe tenerse claro que el mismo debe producir efectos en la cultura de la organización, direccionándola intencionalmente para ganar en capacidades competitivas. No obstante, como mencionan Siti-Nabiha y Scapens (2005, como se citó en Facin Lavarda et al., 2009), y Nor-Aziah y Scapens (2007, como se citó en Facin Lavarda et al., 2009) en sus estudios empíricos, "los hábitos institucionalizados en la 
organización pueden obstaculizar la introducción del nuevo SCG, generar un uso ceremonial del sistema o producir cambios que aquellos que diseñaron las utilidades del nuevo SCG no esperaban” (pág.103).

Un uso ceremonial del sistema de información se entiende como aquel que se realiza como una exigencia rutinaria, mientras el empleado continúa llevando sistemas de información alternos, y no se toman decisiones con base en los reportes de información del sistema. En estos casos no se optimiza el tiempo del empleado ni tampoco se apoyan los procesos administrativos de decisión. Por lo anterior, debe procurarse que se dé un proceso de interiorización por parte de cada empleado para que considere la importancia de alimentar y gestionar correctamente el sistema de información, con este fin es recomendable atender las siguientes consideraciones:

- Los datos que se incorporarán en el sistema de información a implementar deben ser tenidos en cuenta para la toma de decisiones de los niveles medios y estratégicos de la organización; si el personal observa que la información que contiene el sistema no es usada para la gestión perderá el interés en alimentar adecuadamente la aplicación o programa, como resultado se obtendrá información incompleta o mentirosa. Es posible que necesite establecer espacios colectivos para el análisis de información arrojada por el sistema; por ejemplo, una junta semanal o quincenal para revisar el reporte de cartera o para definir las políticas de pago de los clientes.

- Es claro que hay una fuerte predisposición del personal de la Mipyme a creerle más al sistema que a las personas, lo cual permite coordinar con mayor facilidad y con base en datos la ejecución de los procedimientos. No obstante, información errónea puede producir resultados negativos para el negocio, por lo que debe atenderse inmediatamente cualquier imprecisión que presenten los reportes de información, aislando y corrigiendo el problema que normalmente se debe a dificultades con el manejo del sistema por parte de quien lo alimenta.

- Para la implementación y continuidad de la operación del sistema, deben darse procesos de capacitación y formación constante del personal. Estos procesos de capacitación no deben ser únicamente sobre el uso del sistema, sino que deben articularse con los procedimientos de la organización, así como mostrar los resultados esperados de la actividad y 
el uso que se dará al reporte que se construye con la información que se alimenta. En este plano se debe tener en cuenta la "influencia de los conocimientos y habilidades de los agentes implicados en el cambio" (Facin Lavarda et al., 2009, p. 113).

- Deben realizarse revisiones continuas del sistema de información para ajustarlo a los cambios estratégicos y los requeridos por el mejoramiento continuo en la óptica del valor, o ajustar los procedimientos o estructura de la organización para optimizar el funcionamiento de la misma.

- Es importante articular el sistema de incentivos de los operarios a la información almacenada en el sistema, esta es una de las características de éxito en la implementación de sistemas en áreas de producción, aunque también puede aplicarse a otro tipo de tareas. Es importante mencionar que "para que el sistema de incentivos funcione correctamente, se necesita que éste genere confianza entre la empresa y el operario. Una forma de establecer la confianza es cumpliendo con los requisitos de remuneración del rendimiento de los operarios, tal y como previamente se haya establecido" (Facin Lavarda et al., 2009, p. 109).

- Redefinir los manuales de procedimientos antes de realizar la implementación, incorporando las modificaciones en los procesos de decisión y las restricciones que darán las claves de acceso al sistema.

- Verificar si existen cambios en los manuales de funciones o procedimientos al implementar el nuevo sistema. De ser así, deben programarse jornadas de socialización y capacitación sobre los mismos, pues es probable que en algunos casos se tengan que realizar modificaciones sobre las funciones establecidas en los contratos del personal.

- Revisar la carga laboral de cada empleado, pues en algunos casos se pueden dar reducciones significativas de trabajo al incorporar un sistema de información; evaluar la posibilidad de modificar cargos o crear actividades adicionales que generen valor a la organización.

- Migrar la información que está contenida en los sistemas de información actuales de la empresa y verificar los requisitos de información que solicita el nuevo sistema, pues es probable que algunos de los campos 
que se requieren no se encuentren en la organización, por lo que tendrá que aceptar que en los primeros meses de la implementación habrá dificultades al completar la misma.

- Realizar una implementación paulatina del sistema, un procedimiento a la vez, iniciando por aquellos que no repercutirán de forma significativa en la operación del negocio y avanzando hacia los procesos centrales.

- Realizar una implementación iterativa; es decir, a través del procedimiento de socialización, capacitación e implementación de la actividad en el sistema y repetido con cada usuario de la aplicación hasta que no se presenten errores en el manejo del programa ni en la comprensión de la intencionalidad del mismo por parte del empleado.

\section{Conclusiones}

La implementación de un sistema de información contable y administrativa (SICA) es una gran oportunidad para ganar en competitividad a partir de optimizar e innovar en los procesos administrativos, estandarizar los productos de los empleados administrativos de nivel operativo, contar con información para alinear a los gerentes medios o profesionales vitales con la estrategia de negocio, contar con información para evaluar cursos de acción y minimizar los costos de oportunidad que generan las decisiones administrativas.

Por este motivo, la selección o diseño de un SICA se convierte en una actividad estratégica que debe ser liderada por los niveles más altos de la organización. Debe tenerse presente que la compra de un software es más económica que su desarrollo; no obstante, si la selección se realiza erróneamente, cambiar hacia un sistema que esté acorde a las necesidades del negocio o iniciar el desarrollo de una herramienta a la medida aumentará considerablemente los costos del mismo.

La selección de un SICA debe tener en cuenta: 1) la capacidad del sistema para adaptarse o moldear la estructura administrativa; 2) captar información valiosa de la mayor cantidad de procesos en la organización; 3) los aspectos técnicos que influyan en la operación; 4) la capacidad para generar reportes que satisfagan la totalidad de necesidades de información de la organización; y 5) la capacidad del sistema para incidir en la solución 
de problemas en la organización, permitiendo optimizar procedimientos o procesos.

Debe tenerse en cuenta que la implementación del SICA tiene la capacidad de modificar la cultura de la empresa, corrigiendo problemas como la ausencia de cooperación, desconfianza o "vocación" por actuar aisladamente. Por ende, existen dos alternativas para la gerencia al implementarlo, una es adaptar los procesos y procedimientos de acuerdo a la funcionalidad del sistema, y la otra es planear una estructura organizacional óptima y ajustar hasta donde sea posible el diseño del sistema a esta planeación. Por lo anterior, la implementación del SICA debe planearse detalladamente, y para ello se recomienda iniciar con un proceso sistemático de determinación de escenarios de riesgo sobre la funcionalidad del sistema como tal y su influencia en los sistemas de control interno de la empresa.

De igual manera, debe procurarse que la implementación del SICA permita a los empleados la interiorización del uso del mismo, impidiendo el uso ceremonial, ya que en estos casos no se optimiza el tiempo del empleado ni tampoco se apoyan los procesos administrativos de decisión. Por lo tanto, deben observarse recomendaciones tendientes a imbricar el SICA en la subjetividad del empleado a través del entendimiento de los objetivos que se persiguen con la implementación, el uso en la toma de decisiones del sistema y el establecimiento de incentivos basados en la información que este contiene. 


\section{Referencias}

Astudillo Cerón, M. T. (2008). Consideraciones para la selección de sistemas de información contables y administrativos en la Pyme Colombiana. Entramado, 4(2), 52-69.

Barreiro Noa, A. (2003). Los costos de oportunidad de la información y la innovación tecnológica en las empresas. Ciencias de la Información, 34(3), 23-30.

Facin Lavarda, C. E., Ripoll Feliu, V. M., \& Barrachina Palanca, M. (septiembre de 2009). La Interiorización del Cambio de un Sistema Contable de Gestión en la Pequeña Empresa. Revista Contabilidade \& Finanças, 20(51), 101-115.

Gálvez Albarracín, E. J., Riascos Erazo, S. C., \& Contreras Palacios, F. (2014). Influencia de las tecnologías de la información y comunicación en el rendimiento de las micro, pequeñas y medianas empresas colombianas. Estudios Gerenciales, 30(133), 355-364.

González Ramírez, M. R., Gascó Gascó, J. L., \& Llopis Taverner, J. (2010). Razones y Riesgos del Outsourcing de Sistemas de Información: Un Análisis de su Situación y Evolución. Investigaciones Europeas de Dirección y Economía de la Empresa, 16(1), 55-76.

Guerrero Julio, M. L., \& Gómez Flórez, L. C. (2012). Gestión de ries- gos y controles en sistemas de información: del aprendizaje a la transformación organizacional. Estudios Gerenciales, 28, 87-95.

Mora Riapira, E. H., Vera Colina, M., \& Melgarejo Molina, Z. A. (2015). Planificación estratégica y niveles de competitividad de las Mipymes del sector comercio en Bogotá. Estudios Gerenciales, 31(134), 79-87.

Saavedra García, M. L. (junio de 2012). Una Propuesta Para la Determinación de la Competitividad en la PYME Latioamericana. Pensamiento \& Gestión, 33, 93-124.

Vanegas Nieto, N., García Pérez de Lema, D., \& Marín Hernández, S. (diciembre de 2009). Estrategia, Sistemas de Contabilidad de Gestión y Rendimiento en la Industria de la Conserva de Frutas y Vegetales. Cuadernos de Administración, 39(22), 213-233. 